\title{
EXTREMAL MATCHING ENERGY OF COMPLEMENTS OF TREES
}

\author{
Tingzeng $\mathrm{WU}^{1}$ \\ School of Mathematics and Statistics \\ Qinghai Nationalities University \\ Xining, Qinghai 810007, P.R. China \\ e-mail: mathtzwu@163.com \\ WEIGEN YAN \\ School of Sciences, Jimei University \\ Xiamen 361021, P.R. China \\ e-mail: weigenyan@263.net \\ AND \\ HEPING ZHANG \\ School of Mathematics and Statistics, Lanzhou University \\ Lanzhou, Gansu 730000, P.R. China \\ e-mail: zhanghp@lzu.edu.cn
}

\begin{abstract}
Gutman and Wagner proposed the concept of the matching energy which is defined as the sum of the absolute values of the zeros of the matching polynomial of a graph. And they pointed out that the chemical applications of matching energy go back to the 1970s. Let $T$ be a tree with $n$ vertices. In this paper, we characterize the trees whose complements have the maximal, second-maximal and minimal matching energy. Furthermore, we determine the trees with edge-independence number $p$ whose complements have the minimum matching energy for $p=1,2, \ldots,\left\lfloor\frac{n}{2}\right\rfloor$. When we restrict our consideration to all trees with a perfect matching, we determine the trees whose complements have the second-maximal matching energy.
\end{abstract}

Keywords: matching polynomial, matching energy, Hosoya index, energy.

2010 Mathematics Subject Classification: 05C05, 05C35, 92E10.

\footnotetext{
${ }^{1}$ Corresponding author.
} 


\section{REFERENCES}

[1] J. Aihara, A new definition of Dewar-type resonance energies, J. Amer. Chem. Soc. 98 (1976) 2750-2758.

doi:10.1021/ja00426a013

[2] L. Chen and Y. Shi, The maximal matching energy of tricyclic graphs, MATCH Commun. Math. Comput. Chem. 73 (2015) 105-119.

[3] L. Chen, J. Liu and Y. Shi, Matching energy of unicyclic and bicyclic graphs with a given diameter, Complexity 21 (2015) 224-238. doi:10.1002/cplx.21599

[4] D. Cvetković, M. Doob, I. Gutman and A. Torgašev, Recent Results in the Theory of Graph Spectra (North-Holland, Amsterdam, 1988).

[5] M.J.S. Dewar, The Molecular Orbital Theory of Organic Chemistry (McGraw-Hill, New York, 1969).

[6] E.J. Farrell, An introduction to matching polynomials, J. Combin. Theory Ser. B 27 (1979) 75-86. doi:10.1016/0095-8956(79)90070-4

[7] C.D. Godsil, Algebraic Combinatorics (Chapman and Hall, New York, 1993).

[8] C.D. Godsil and I. Gutman, On the theory of the matching polynomial, J. Graph Theory 5 (1981) 137-144. doi:10.1002/jgt.3190050203

[9] I. Gutman, The matching polynomial, MATCH Commun. Math. Comput. Chem. 6 (1979) 75-91.

[10] I. Gutman and S. Wagner, The matching energy of a graph, Discrete Appl. Math. 160 (2012) 2177-2187. doi:10.1016/j.dam.2012.06.001

[11] I. Gutman, The energy of a graph: old and new results, in: Algebraic Combinatorics and Applications, A. Betten, A. Kohnert, R. Laue, A. Wassermann (Ed(s)), (Springer-Verlag, Berlin, 2001) 196-211. doi:10.1007/978-3-642-59448-9_13

[12] I. Gutman, X. Li and J. Zhang, Graph energy, in: Analysis of Complex Networks From Biology to Linguistics, M. Dehmer, F. Emmert-Streib (Ed(s)), (Wiley-VCH, Weinheim, 2009) 145-174. doi:10.1002/9783527627981.ch7

[13] S. Ji, X. Li and Y. Shi, Extremal matching energy of bicyclic graphs, MATCH Commun. Math. Comput. Chem. 70 (2013) 697-706.

[14] H. Li, Y. Zhou and L. Su, Graphs with extremal matching energies and prescribed parameters, MATCH Commun. Math. Comput. Chem. 72 (2014) 239-248.

[15] S. Li and W. Yan, The matching energy of graphs with given parameters, Discrete Appl. Math. 162 (2014) 415-420. doi:10.1016/j.dam.2013.09.014 
[16] X. Li, Y. Shi and I. Gutman, Graph Energy (Springer, New York, 2012). doi:10.1007/978-1-4614-4220-2

[17] L. Lovász, Combinatorial Problems and Exercises, Second Edition (Budapest, Akadémiai Kiadó, 1993).

[18] D.B. West, Introduction to Graph Theory, Second Edition (Pearson Education, Singapore, 2001).

[19] T. Wu, On the maximal matching energy of graphs, J. East China Norm. Univ. 1 (2015) 136-141.

[20] K. Xu, Z. Zheng and K.C. Das, Extremal t-apex trees with respect to matching energy, Complexity (2015), in press. doi: $10.1002 /$ cplx.21651

[21] K. Xu, K.C. Das and Z. Zheng, The minimum matching energy of $(n, m)$-graphs with a given matching number, MATCH Commun. Math. Comput. Chem. 73 (2015) 93-104.

[22] W. Yan, Y. Yeh and F. Zhang, Ordering the complements of trees by the number of maximum matchings, J. Quan. Chem. 1055 (2005) 131-141. doi:10.1002/qua.20688

Received 1 February 2015

Revised 9 August 2015

Accepted 9 August 2015 